\title{
2 - Variable AQCQ - Functional equation
}

\author{
M. Arunkumar ${ }^{1 *}$, S. Hema Latha ${ }^{2}$, E. Sathya ${ }^{3}$ \\ ${ }^{1,3}$ Department of Mathematics, Government Arts College, Tiruvannamalai - 606 603, TamilNadu, India \\ 2 Department of Mathematics, Annai Veilankanni's College of Arts and Science, Chennai - 600 015, TamilNadu, India \\ *Corresponding author E-mail: annarun2002@yahoo.co.in
}

Copyright (C)2015 Arunkumar et. all. This is an open access article distributed under the Creative Commons Attribution License, which permits unrestricted use, distribution, and reproduction in any medium, provided the original work is properly cited.

\begin{abstract}
In this paper, the authors obtain the general solution and generalized Ulam - Hyers stability of a 2 - variable AQCQ functional equation

$$
\begin{aligned}
g(x+2 y, u+2 v)+g(x-2 y, u-2 v)= & 4[g(x+y, u+v)+g(x-y, u-v)]-6 g(x, u) \\
& +g(2 y, 2 v)+g(-2 y,-2 v)-4 g(y, v)-4 g(-y,-v)
\end{aligned}
$$

using Hyers direct method. Counter examples for non stability is also discussed.

Keywords: Additive functional equations, quadratic functional equations, cubic functional equations, quartic functional equations, Mixed type functional equations, Ulam - Hyers stability, Ulam - Hyers - Rassias stability, Ulam - Gavruta - Rassias stability, Ulam JRassias stability.
\end{abstract}

\section{Introduction}

The study of stability problems for functional equations is related to a question of Ulam [23] concerning the stability of group homomorphisms and affirmatively answered for Banach spaces by Hyers [12]. It was further generalized and excellent results obtained by number of authors [2, 7, 18, 21, 19].

Over the last seven decades, the above problem was tackled by numerous authors and its solutions via various forms of functional equations like additive, quadratic, cubic, quartic, mixed type functional equations which involves only these types of functional equations were discussed. We refer the interested readers for more information on such problems to the monographs $[1,6,8,9,11,13,14,15,22,24,25,26]$

The general solution and the generalized Hyers-Ulam-Rassias stability of the generalized mixed type of functional equation

$$
\begin{aligned}
f(x+a y)+f(x-a y) & =a^{2}[f(x+y)+f(x-y)]+2\left(1-a^{2}\right) f(x) \\
& +\frac{\left(a^{4}-a^{2}\right)}{12}[f(2 y)+f(-2 y)-4 f(y)-4 f(-y)] .
\end{aligned}
$$

for fixed integers $a$ with $a \neq 0, \pm 1$ having solution additive, quadratic, cubic and quartic was discussed by K. Ravi et. al., [20]. Its generalized Ulam-Hyers stability in multi-Banach spaces and non-Archimedean normed spaces via fixed point approach was respectively investigated by T.Z. Xu et. al [24, 26]. 
Recently, M. Arunkumar et al., [3] first time introduced and investigated the solution and generalized Ulam-Hyers stability of a 2 - variable $\mathrm{AC}$ - mixed type functional equation

$f(2 x+y, 2 z+w)-f(2 x-y, 2 z-w)=4[f(x+y, z+w)-f(x-y, z-w)]-6 f(y, w)$

having solutions

$f(x, y)=a x+b y$

and

$f(x, y)=a x^{3}+b x^{2} y+c x y^{2}+d y^{3}$

in Banach space via direct and fixed point approach.

Very recently, Choonkil Park and Jung Rye Lee [17] proved the Hyers - Ulam stability of the following additive - quadratic - cubic - quartic functional equation

$f(x+2 y)+f(x-2 y)=4 f(x+y)+4 f(x-y)-6 f(x)+f(2 y)+f(-2 y)-4 f(y)-4 f(-y)$

in paranormed spaces.

In this paper, the authors obtain the general solution and generalized Ulam - Hyers stability of a 2 - variable AQCQ functional equation

$$
\begin{aligned}
g(x+2 y, u+2 v)+g(x-2 y, u-2 v)= & 4[g(x+y, u+v)+g(x-y, u-v)]-6 g(x, u) \\
& +g(2 y, 2 v)+g(-2 y,-2 v)-4 g(y, v)-4 g(-y,-v)
\end{aligned}
$$

having solutions

$g(x, y)=a x+b y$,

$g(x, y)=a x^{2}+b x y+c y^{2}$,

$g(x, y)=a x^{3}+b x^{2} y+c x y^{2}+d y^{3}$,

$g(x, y)=a x^{4}+b x^{3} y+c x^{2} y^{2}+d x y^{3}+e y^{4}$.

In Section 2, we present the general solution of the functional equation (5). The generalized Ulam-Hyers stability using Hyers direct method for odd, even and mixed cases are respectively discussed in Sections 3, 4, and 5 . In Sections 6 and 7, counter examples for non stability for odd and even case are respectively given.

\section{General solution}

In this section, we present the solution of the functional equation (5). Through out this section let $G$ and $H$ be real vector spaces.

Lemma 2.1 If $g: G^{2} \rightarrow H$ be an odd mapping satisfying (5) and let $a: G^{2} \rightarrow H$ be a mapping given by

$a(u, u)=g(2 u, 2 u)-8 g(u, u)$

for all $u \in G$ then

$a(2 u, 2 u)=2 a(u, u)$

for all $u \in G$ such that $a$ is additive.

Proof. Using oddness of $g$ in (5), we arrive

$g(x+2 y, u+2 v)+g(x-2 y, u-2 v)=4[g(x+y, u+v)+g(x-y, u-v)]-6 g(x, u)$

for all $x, y, u, v \in G$. Letting $(x, y, u, v)$ by $(0,0,0,0)$ in (12), we get

$g(0,0)=0$. 
Replacing $(x, y, z, w)$ by $(u, u, u, u)$ in $(3.2)$, we arrive

$g(3 u, 3 u)=4 g(2 u, 2 u)-5 g(u, u)$

for all $u \in G$. Again replacing $(x, y, u, v)$ by $(2 u, u, 2 u, u)$ in (12) and using (13), (14), we have

$g(4 u, 4 u)=4 g(3 u, 3 u)-6 g(2 u, 2 u)+4 g(u, u)$

for all $u \in G$. Using (14) in (15), we get

$g(4 u, 4 u)=10 g(2 u, 2 u)-16 g(u, u)$

for all $u \in G$. From (10), we establish

$a(2 u, 2 u)-2 a(u, u)=g(4 u, 4 u)-10 g(2 u, 2 u)+16 g(u, u)$

for all $u \in G$. Using (16) in (17), we desired our result.

Lemma 2.2 If $g: G^{2} \rightarrow H$ be an even mapping satisfying (5) and let $c: G^{2} \rightarrow H$ be a mapping given by

$c(u, u)=g(2 u, 2 u)-2 g(u, u)$

for all $u \in G$ then

$c(2 u, 2 u)=8 c(u, u)$

for all $u \in G$ such that $c$ is cubic.

Proof. It follows from (19) that

$c(2 u, 2 u)-8 c(u, u)=g(4 u, 4 u)-10 g(2 u, 2 u)+16 g(u, u)$

for all $x \in U$. Using (16) in (20), we desired our result.

Lemma 2.3 If $g: G^{2} \rightarrow H$ be an even mapping satisfying (5) and let $q_{2}: G^{2} \rightarrow H$ be a mapping given by

$q_{2}(u, u)=g(2 u, 2 u)-16 g(u, u)$

for all $u \in G$ then

$q_{2}(2 u, 2 u)=4 q_{2}(u, u)$

for all $u \in G$ such that $q_{2}$ is quadratic.

Proof.

Using evenness of $g$ in (5), we arrive

$g(x+2 y, u+2 v)+g(x-2 y, u-2 v)$
$\quad=4[g(x+y, u+v)+g(x-y, u-v)]-6 g(x, u)+2 g(2 y, 2 v)-8 g(y, v)$

for all $x, y, u, v \in G$. Letting $(x, y, u, v)$ by $(0,0,0,0)$ in $(23)$, we get

$g(0,0)=0$.

Replacing $(x, y, z, w)$ by $(u, u, u, u)$ in $(23)$, we arrive

$g(3 u, 3 u)=6 g(2 u, 2 u)-15 g(u, u)$

for all $u \in G$. Again replacing $(x, y, u, v)$ by $(2 u, u, 2 u, u)$ in (23) and using (24), (25), we have

$g(4 u, 4 u)=4 g(3 u, 3 u)-4 g(2 u, 2 u)-4 g(u, u)$

for all $u \in G$. Using (25) in (26), we get

$g(4 u, 4 u)=20 g(2 u, 2 u)-64 g(u, u)$

for all $u \in G$. From (21), we establish

$q_{2}(2 u, 2 u)-4 q_{2}(u, u)=g(4 u, 4 u)-20 g(2 u, 2 u)+64 g(u, u)$

for all $u \in G$. Using (27) in (28), we desired our result. 
Lemma 2.4 If $g: G^{2} \rightarrow H$ be an even mapping satisfying (5) and let $q_{4}: G^{2} \rightarrow H$ be a mapping given by

$q_{4}(u, u)=g(2 u, 2 u)-4(u, u)$

for all $u \in G$ then

$q_{4}(2 u, 2 u)=16 q_{4}(u, u)$

for all $u \in G$ such that $q_{4}$ is quartic.

Proof. It follows from (30) that

$q_{4}(2 u, 2 u)-4 q_{4}(u, u)=g(4 u, 4 u)-20 g(2 u, 2 u)+64 g(u, u)$

for all $x \in U$. Using (27) in (31), we desired our result.

Remark 2.5 If $g: G^{2} \rightarrow H$ be a mapping satisfying (5) and let $a, q_{2}, c, q_{4}: G^{2} \rightarrow H$ be a mapping defined in (10), (18), (21) and (29) then

$g(u, u)=\frac{1}{12}\left(q_{4}(u, u)-q_{2}(u, u)\right)$

and

$g(u, u)=\frac{1}{6}(c(u, u)-a(u, u))$

for all $u \in G$.

Lemma 2.6 If $g: G^{2} \rightarrow H$ be a mapping satisfying (5) and let $f: G \rightarrow H$ be a mapping given by

$f(u)=g(u, u)$

for all $u \in G$, then $f$ is (4) for all $x, y, u, v \in G$.

Proof. From (5) and (34), we get

$$
\begin{aligned}
f(x+ & 2 y)+f(x-2 y) \\
& =g(x+2 y, x+2 y)+g(x-2 y, x-2 y) \\
& =4[g(x+y, x+y)+g(x-y, x-y)]-6 g(x, x)+g(2 y, 2 y)+g(-2 y,-2 y)-4 g(y, y)-4 g(-y,-y) \\
& =4[f(x+y)+f(x-y)]-6 f(x)+f(2 y)+f(-2 y)-4 f(y)-4 f(-y)
\end{aligned}
$$

for all $x, y \in G$.

Hereafter, through out this paper, let we consider $G$ be a normed space and $H$ be a Banach space. Define a mapping $D g: G^{2} \rightarrow H$ by

$$
\begin{aligned}
D g(x, y, u, v)= & g(x+2 y, u+2 v)+g(x-2 y, u-2 v)-4[g(x+y, u+v)+g(x-y, u-v)] \\
& +6 g(x, u)-g(2 y, 2 v)-g(-2 y,-2 v)+4 g(y, v)+4 g(-y,-v)
\end{aligned}
$$

for all $x, y, u, v \in G$.

\section{Stability results: Odd case}

In this section, we investigate the generalized Ulam-Hyers stability of the functional equation (5) for odd case.

Theorem 3.1 Let $j= \pm 1$. Let $D g: G^{2} \rightarrow H$ be an odd mapping for which there exist a function $\alpha: G^{4} \rightarrow[0, \infty)$ with the condition

$\lim _{n \rightarrow \infty} \frac{1}{2^{n j}} \alpha\left(2^{n j} x, 2^{n j} y, 2^{n j} u, 2^{n j} v\right)=0$ 
such that the functional inequality

$\|D g(x, y, u, v)\| \leq \alpha(x, y, u, v)$

for all $x, y, u, v \in G$. Then there exists a unique 2-variable additive mapping $A(u, u): G^{2} \rightarrow H$ satisfying the functional equation (5) and

$\|g(2 u, 2 u)-8 g(u, u)-A(u, u)\| \leq \frac{1}{2} \sum_{k=\frac{1-j}{2}}^{\infty} \frac{\beta\left(2^{k j} u\right)}{2^{k j}}$

for all $u \in G$, where $\beta\left(2^{k j} u\right)$ and $A(u, u)$ are defined by

$\beta\left(2^{k j} u\right)=4 \alpha\left(2^{k j} u, 2^{k j} u, 2^{k j} u, 2^{k j} u\right)+\alpha\left(2^{(k+1) j} u, 2^{k j} u, 2^{(k+1) j} u, 2^{k j} u\right)$

$A(u, u)=\lim _{n \rightarrow \infty} \frac{1}{2^{n j}}\left(g\left(2^{(n+1) j} u, 2^{(n+1) j} u\right)-8 g\left(2^{n j} u, 2^{n j} u\right)\right)$

for all $u \in G$.

Proof. Assume $j=1$. Letting $(x, y, u, v)$ by $(u, u, u, u)$ in (36) and using oddness of $g$, we obtain

$\|g(3 u, 3 u)-4 g(2 u, 2 u)+5 g(u, u)\| \leq \alpha(u, u, u, u)$

for all $u \in G$. Replacing $(x, y, z, w)$ by $(2 u, u, 2 u, u)$ in $(36)$, we get

$\|g(4 u, 4 u)-4 g(3 u, 3 u)+6 g(2 u, 2 u)-4 g(u, u)\| \leq \alpha(2 u, u, 2 u, u)$

for all $u \in G$. Now, from (40) and (41), we have

$$
\begin{aligned}
& \|g(4 u, 4 u)-10 g(2 u, 2 u)+16 g(u, u)\| \\
& \quad \leq 4\|g(3 u, 3 u)-4 g(2 u, 2 u)+5 g(u, u)\|+\|g(4 u, 4 u)-4 g(3 u, 3 u)+6 g(2 u, 2 u)-4 g(u, u)\| \\
& \quad \leq 4 \alpha(u, u, u, u)+\alpha(2 u, u, 2 u, u)
\end{aligned}
$$

for all $u \in G$. From (42), we arrive

$\|g(4 u, 4 u)-10 g(2 u, 2 u)+16 g(u, u)\| \leq \beta(u)$

where

$\beta(u)=4 \alpha(u, u, u, u)+\alpha(2 u, u, 2 u, u)$

for all $u \in G$. It is easy to see from (43) that

$\|g(4 u, 4 u)-8 g(2 u, 2 u)-2(g(2 u, 2 u)-8 g(u, u))\| \leq \beta(u)$

for all $u \in G$. Using (10) in (45), we obtain

$\|a(2 u, 2 u)-2 a(u, u)\| \leq \beta(u)$

for all $u \in G$. From (46), we arrive

$\left\|\frac{a(2 u, 2 u)}{2}-a(u, u)\right\| \leq \frac{\beta(u)}{2}$

for all $u \in G$. Now replacing $u$ by $2 u$ and dividing by 2 in (47), we get

$\left\|\frac{a\left(2^{2} u, 2^{2} u\right)}{2^{2}}-\frac{a(2 u, 2 u)}{2}\right\| \leq \frac{\beta(2 u)}{2^{2}}$ 
for all $u \in G$. From (47) and (48), we obtain

$$
\begin{aligned}
\left\|\frac{a\left(2^{2} u, 2^{2} u\right)}{2^{2}}-a(u, u)\right\| & \leq\left\|\frac{a(2 u, 2 u)}{2}-a(u, u)\right\|+\left\|\frac{a\left(2^{2} u, 2^{2} u\right)}{2^{2}}-\frac{a(2 u, 2 u)}{2}\right\| \\
& \leq \frac{1}{2}\left[\beta(u)+\frac{\beta(2 u)}{2}\right]
\end{aligned}
$$

for all $u \in G$. Proceeding further and using induction on a positive integer $n$, we get

$$
\begin{aligned}
\left\|\frac{a\left(2^{n} u, 2^{n} u\right)}{2^{n}}-a(u, u)\right\| & \leq \frac{1}{2} \sum_{k=0}^{n-1} \frac{\beta\left(2^{k} u\right)}{2^{k}} \\
& \leq \frac{1}{2} \sum_{k=0}^{\infty} \frac{\beta\left(2^{k} u\right)}{2^{k}}
\end{aligned}
$$

for all $u \in G$. In order to prove the convergence of the sequence

$$
\left\{\frac{a\left(2^{n} u, 2^{n} u\right)}{2^{n}}\right\}
$$

replacing $u$ by $2^{m} u$ and dividing by $2^{m}$ in (50), for any $m, n>0$, we deduce

$$
\begin{aligned}
\left\|\frac{a\left(2^{n+m} u, 2^{n+m} u\right)}{2^{(n+m)}}-\frac{a\left(2^{m} u, 2^{m} u\right)}{2^{m}}\right\| & =\frac{1}{2^{m}}\left\|\frac{a\left(2^{n} \cdot 2^{m} u, 2^{n} \cdot 2^{m} u\right)}{2^{n}}-a\left(2^{m} u, 2^{m} u\right)\right\| \\
& \leq \frac{1}{2} \sum_{k=0}^{\infty} \frac{\beta\left(2^{k+m} u\right)}{2^{k+m}} \\
& \rightarrow 0 \text { as } m \rightarrow \infty
\end{aligned}
$$

for all $u \in G$. This shows that the sequence $\left\{\frac{a\left(2^{n} u, 2^{n} u\right)}{2^{n}}\right\}$ is Cauchy sequence. Since $H$ is complete, there exists a mapping $A(u, u): G^{2} \rightarrow H$ such that

$$
A(u, u)=\lim _{n \rightarrow \infty} \frac{a\left(2^{n} u, 2^{n} u\right)}{2^{n}} \forall u \in G .
$$

Letting $n \rightarrow \infty$ in (50) and using (10), we see that (37) holds for all $u \in G$. To show that $A$ satisfies (5), replacing $(x, y, u, v)$ by $\left(2^{n} x, 2^{n} y, 2^{n} u, 2^{n} v\right)$ and dividing by $2^{n}$ in (36), we obtain

$\frac{1}{2^{n}}\left\|D g\left(2^{n} x, 2^{n} y, 2^{n} u, 2^{n} v\right)\right\| \leq \frac{1}{2^{n}} \alpha\left(2^{n} x, 2^{n} y, 2^{n} u, 2^{n} v\right)$

for all $x, y, u, v \in G$. Letting $n \rightarrow \infty$ in the above inequality and using the definition of $A(u, u)$, we see that

$D A(x, y, u, v)=0$.

Hence $A$ satisfies (5) for all $x, y, u, v \in G$. To prove $A$ is unique 2 -variable additive function satisfying (5), we let $B(u, u)$ be another 2 -variable additive mapping satisfying (5) and (37), then

$$
\begin{aligned}
\|A(u, u)-B(u, u)\|= & \frac{1}{2^{n}}\left\|A\left(2^{n} u, 2^{n} u\right)-B\left(2^{n} u, 2^{n} u\right)\right\| \\
\leq & \frac{1}{2^{n}}\left\{\left\|A\left(2^{n} u, 2^{n} u\right)-g\left(2^{n+1} u, 2^{n+1} u\right)+8 g\left(2^{n} u, 2^{n} u\right)\right\|\right. \\
& \left.+\left\|g\left(2^{n+1} u, 2^{n+1} u\right)-8 g\left(2^{n} u, 2^{n} u\right)-B\left(2^{n} u, 2^{n} u\right)\right\|\right\} \\
\leq & \sum_{k=0}^{\infty} \frac{\beta\left(2^{k+n} u\right)}{2^{(k+n)}} \\
& \rightarrow 0 \text { as } n \rightarrow \infty
\end{aligned}
$$

for all $u \in G$. Hence $A$ is unique.

For $j=-1$, we can prove a similar stability result. This completes the proof of the theorem.

The following Corollary is an immediate consequence of Theorem 3.1 concerning the Ulam-Hyers, Ulam-HyersRassias, Ulam-Gavruta-Rassias and Ulam-JRassias stabilities of (5). 
Corollary 3.2 Let $D g: G^{2} \rightarrow H$ be a mapping and there exits real numbers $\rho$ and $s$ such that

$$
\begin{aligned}
& \text { \|Dg(x,y,u,v)\| } \\
& \leq\left\{\begin{array}{llll}
(i) & \rho, & & \\
(i i) & \rho\left\{\|x\|^{s}+\|y\|^{s}+\|u\|^{s}+\|v\|^{s}\right\}, & s<1 & \text { or } \quad s>1 ; \\
(\text { iii }) & \rho\|x\|^{s}\|y\|^{s}\|u\|^{s}\|v\|^{s}, & s<\frac{1}{4} & \text { or } \quad s>\frac{1}{4} \\
(\text { iv }) & \rho\left\{\|x\|^{s}\|y\|^{s}\|u\|^{s}\|v\|^{s}+\left\{\|x\|^{4 s}+\|y\|^{4 s}+\|u\|^{4 s}+\|v\|^{4 s}\right\}\right\}, & s<\frac{1}{4} & \text { or } \quad s>\frac{1}{4}
\end{array}\right.
\end{aligned}
$$

for all $x, y, u, v \in G$, then there exists a unique 2- variable additive function $A(u, u): G^{2} \rightarrow H$ such that

$$
\|g(2 u, 2 u)-8 g(u, u)-A(u, u)\| \leq \begin{cases}\text { (i) } & 5 \rho, \\ \text { (ii) } & \frac{\left(18+2^{s+1}\right) \rho\|u\|^{s}}{\left|2-2^{s}\right|} \\ \text { (iii) } & \frac{\left(4+2^{2 s}\right) \rho\|u\|^{4 s}}{\left|2-2^{4 s}\right|} \\ \text { (iv) } & \left(\frac{22+2^{2 s}+2^{4 s+1}}{\left|2-2^{4 s}\right|}\right) \rho\|u\|^{4 s}\end{cases}
$$

for all $u \in G$.

Theorem 3.3 Let $j= \pm 1$. Let $D g: G^{2} \rightarrow H$ be an odd mapping for which there exist a function $\alpha: G^{4} \rightarrow[0, \infty)$ with the condition

$\lim _{n \rightarrow \infty} \frac{1}{8^{n j}} \alpha\left(2^{n j} x, 2^{n j} y, 2^{n j} u, 2^{n j} v\right)=0$

such that the functional inequality

$\|D g(x, y, u, v)\| \leq \alpha(x, y, u, v)$

for all $x, y, u, v \in G$. Then there exists a unique 2-variable cubic mapping $C(u, u): G^{2} \rightarrow H$ satisfying the functional equation (5) and

$\|g(2 u, 2 u)-2 g(u, u)-C(u, u)\| \leq \frac{1}{8} \sum_{k=\frac{1-j}{2}}^{\infty} \frac{\beta\left(2^{k j} u\right)}{8^{k j}}$

for all $u \in G$, where $\beta\left(2^{k j} u\right)$ and $C(u, u)$ are defined by

$\beta\left(2^{k j} u\right)=4 \alpha\left(2^{k j} u, 2^{k j} u, 2^{k j} u, 2^{k j} u\right)+\alpha\left(2^{(k+1) j} u, 2^{k j} u, 2^{(k+1) j} u, 2^{k j} u\right)$

$C(u, u)=\lim _{n \rightarrow \infty} \frac{1}{8^{n j}}\left(g\left(2^{(n+1) j} u, 2^{(n+1) j} u\right)-2 g\left(2^{n j} u, 2^{n j} u\right)\right)$

for all $u \in G$.

Proof. It is easy to see from (43) that

$\|g(4 u, 4 u)-2 g(2 u, 2 u)-8(g(2 u, 2 u)-2 g(u, u))\| \leq \beta(u)$

for all $u \in G$. Using (18) in (58), we obtain

$\|c(2 u, 2 u)-8 c(u, u)\| \leq \beta(u)$

for all $u \in G$. The rest of proof is similar tracing to that of Theorem 3.1.

The following Corollary is an immediate consequence of Theorem 3.3 concerning the Ulam-Hyers, Ulam-HyersRassias, Ulam-Gavruta-Rassias and Ulam-JRassias stabilities of (5). 
Corollary 3.4 Let $D g: G^{2} \rightarrow H$ be a mapping and there exits real numbers $\rho$ and $s$ such that

$\|D g(x, y, u, v)\|$

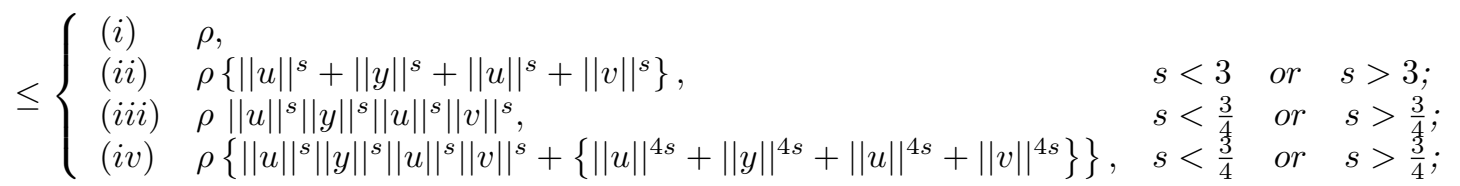

for all $x, y, u, v \in G$, then there exists a unique 2-variable cubic function $C(u, u): G^{2} \rightarrow H$ such that

$$
\|g(2 u, 2 u)-2 g(u, u)-C(u, u)\| \leq \begin{cases}\text { (i) } & \frac{5 \rho}{7}, \\ \text { (ii) } & \frac{\left(18+2^{s+1}\right) \rho\|u\|^{s}}{\left|8-2^{s}\right|} \\ \text { (iii) } & \frac{\left(4+2^{2 s}\right) \rho\|u\|^{4 s}}{\left|8-2^{4 s}\right|} \\ \text { (iv) } & \left(\frac{22+2^{2 s}+2^{4 s+1}}{\left|8-2^{4 s}\right|}\right) \rho\|u\|^{4 s}\end{cases}
$$

for all $u \in G$.

Theorem 3.5 Let $j= \pm 1$. Let $D g: G^{2} \rightarrow H$ be a mapping for which there exist a function $\alpha: G^{4} \rightarrow[0, \infty)$ with the condition given in (35) and (53) respectively, such that the functional inequality

$\|D g(x, y, u, v)\| \leq \alpha(x, y, u, v)$

for all $x, y, u, v \in G$. Then there exists a unique 2-variable additive mapping $A(u, u): G^{2} \rightarrow H$ and a unique 2-variable cubic mapping $C(u, u): G^{2} \rightarrow H$ satisfying the functional equation (5) and

$\|g(u, u)-A(u, u)-C(u, u)\| \leq \frac{1}{6}\left\{\frac{1}{2} \sum_{k=\frac{1-j}{2}}^{\infty} \frac{\beta\left(2^{k j} u\right)}{2^{k j}}+\frac{1}{8} \sum_{k=\frac{1-j}{2}}^{\infty} \frac{\beta\left(2^{k j} u\right)}{8^{k j}}\right\}$

for all $u \in G$, where $\beta\left(2^{k j} u\right), A(u, u)$ and $C(u, u)$ are respectively defined in (38), (39) and (57) for all $u \in G$.

Proof. By Theorems 3.1 and 3.3, there exists a unique 2-variable additive function $A_{1}(u, u): G^{2} \rightarrow H$ and a unique 2-variable cubic function $C_{1}(u, u): G^{2} \rightarrow H$ such that

$\left\|g(2 u, 2 u)-8 g(u, u)-A_{1}(u, u)\right\| \leq \frac{1}{2} \sum_{k=\frac{1-j}{2}}^{\infty} \frac{\beta\left(2^{k j} x\right)}{2^{k j}}$

and

$\left\|g(2 u, 2 u)-2 g(u, u)-C_{1}(u, u)\right\| \leq \frac{1}{8} \sum_{k=\frac{1-j}{2}}^{\infty} \frac{\beta\left(2^{k j} x\right)}{8^{k j}}$

for all $u \in G$. Now from (64) and (65), one can see that

$$
\begin{aligned}
\| g & (u, u)+\frac{1}{6} A_{1}(u, u)-\frac{1}{6} C_{1}(u, u) \| \\
& =\left\|\left\{-\frac{g(2 u, 2 u)}{6}+\frac{8 g(u, u)}{6}+\frac{A_{1}(u, u)}{6}\right\}+\left\{\frac{g(2 u, 2 u)}{6}-\frac{2 g(u, u)}{6}-\frac{C_{1}(u, u)}{6}\right\}\right\| \\
& \leq \frac{1}{6}\left\{\left\|g(2 u, 2 u)-8 g(u, u)-A_{1}(u, u)\right\|+\left\|g(2 u, 2 u)-2 g(u, u)-C_{1}(u, u)\right\|\right\} \\
& \leq \frac{1}{6}\left\{\frac{1}{2} \sum_{k=\frac{1-j}{2}}^{\infty} \frac{\beta\left(2^{k j} x\right)}{2^{k j}}+\frac{1}{8} \sum_{k=\frac{1-j}{2}}^{\infty} \frac{\beta\left(2^{k j} x\right)}{8^{k j}}\right\}
\end{aligned}
$$

for all $u \in U$. Thus we obtain (65) by defining $A(u, u)=\frac{-1}{6} A_{1}(u, u)$ and $C(u, u)=\frac{1}{6} C_{1}(u, u)$, where $\beta\left(2^{k j} u\right)$, $A(u, u)$ and $C(u, u)$ are respectively defined in (38), (39) and (57) for all $u \in G$.

The following corollary is the immediate consequence of Theorem 3.5, using Corollaries 3.2 and 3.4 concerning the Ulam-Hyers, Ulam-Hyers-Rassias, Ulam-Gavruta-Rassias and Ulam-JRassias stabilities of (5). 
Corollary 3.6 Let $D g: G^{2} \rightarrow H$ be a mapping and there exits real numbers $\rho$ and $s$ such that

$\|D g(x, y, u, v)\|$

$$
\leq\left\{\begin{array}{lll}
(i) & \rho, & \\
(i i) & \rho\left\{\|x\|^{s}+\|y\|^{s}+\|u\|^{s}+\|v\|^{s}\right\}, & s \neq 1,3 \\
(\text { iii }) & \rho\|x\|\left\|^{s}\right\| y\left\|^{s}\right\| u\left\|^{s}\right\| v \|^{s}, & s \neq \frac{1}{4}, \frac{3}{4} \\
(\text { iv }) & \rho\left\{\|x\|^{s}\|y\|^{s}\|u\|^{s}\|v\|^{s}+\left\{\|x\|^{4 s}+\|y\|^{4 s}+\|u\|^{4 s}+\|v\|^{4 s}\right\}\right\}, & s \neq \frac{1}{4}, \frac{3}{4}
\end{array}\right.
$$

for all $x, y, u, v \in G$, then there exists a unique 2-variable additive mapping $A(u, u): G^{2} \rightarrow H$ and a unique 2-variable cubic mapping $C(u, u): G^{2} \rightarrow H$ such that

$$
\begin{aligned}
& \|g(u, u)-A(u, u)-C(u, u)\| \\
& \quad \leq \begin{cases}(i) & \frac{5 \rho}{6}\left(1+\frac{1}{7}\right), \\
(i i) & \frac{\left(18+2^{s+1}\right)}{6}\left(\frac{1}{\left|2-2^{s}\right|}+\frac{1}{\left|8-2^{s}\right|}\right) \rho\|u\|^{s}, \\
(i i i) & \frac{\left(4+2^{2 s}\right)}{6}\left(\frac{1}{\left|2-2^{4 s}\right|}+\frac{1}{\mid 8-2^{4 s}}\right) \rho\|u\|^{4 s} \\
(i v) & \frac{\left(22+2^{2 s}+2^{4 s+1}\right)}{6}\left(\frac{1}{\mid 2-2^{4 s \mid}}+\frac{1}{\mid 8-2^{4 s \mid}}\right) \rho\|u\|^{4 s}\end{cases}
\end{aligned}
$$

for all $u \in G$.

\section{Stability results: Even case}

In this section, we present the generalized Ulam-Hyers stability of the functional equation (5) for even case.

Theorem 4.1 Let $j= \pm 1$. Let $D g: G^{2} \rightarrow H$ be an even mapping for which there exist a function $\alpha: G^{4} \rightarrow[0, \infty)$ with the condition

$\lim _{n \rightarrow \infty} \frac{1}{4^{n j}} \alpha\left(2^{n j} x, 2^{n j} y, 2^{n j} u, 2^{n j} v\right)=0$

such that the functional inequality

$\|D g(x, y, u, v)\| \leq \alpha(x, y, u, v)$

for all $x, y, u, v \in G$. Then there exists a unique 2-variable quadratic mapping $Q_{2}(u, u): G^{2} \rightarrow H$ satisfying the functional equation (5) and

$\left\|g(2 u, 2 u)-16 g(u, u)-Q_{2}(u, u)\right\| \leq \frac{1}{4} \sum_{k=\frac{1-j}{2}}^{\infty} \frac{\beta\left(2^{k j} u\right)}{4^{k j}}$

for all $u \in G$, where $\beta\left(2^{k j} u\right)$ and $Q(u, u)$ are defined by

$\beta\left(2^{k j} u\right)=4 \alpha\left(2^{k j} u, 2^{k j} u, 2^{k j} u, 2^{k j} u\right)+\alpha\left(2^{(k+1) j} u, 2^{k j} u, 2^{(k+1) j} u, 2^{k j} u\right)$

$Q_{2}(u, u)=\lim _{n \rightarrow \infty} \frac{1}{4^{n j}}\left(g\left(2^{(n+1) j} u, 2^{(n+1) j} u\right)-16 g\left(2^{n j} u, 2^{n j} u\right)\right)$

for all $u \in G$.

Proof. Assume $j=1$. Letting $(x, y, u, v)$ by $(u, u, u, u)$ in (69) and using evenness of $g$, we obtain

$\|g(3 u, 3 u)-6 g(2 u, 2 u)+15 g(u, u)\| \leq \alpha(u, u, u, u)$

for all $u \in G$. Replacing $(x, y, z, w)$ by $(2 u, u, 2 u, u)$ in (69), we get

$\|g(4 u, 4 u)-4 g(3 u, 3 u)+4 g(2 u, 2 u)+4 g(u, u)\| \leq \alpha(2 u, u, 2 u, u)$ 
for all $u \in G$. Now, from (73) and (74), we have

$$
\begin{aligned}
& \|g(4 u, 4 u)-20 g(2 u, 2 u)+64 g(u, u)\| \\
& \quad \leq 4\|g(3 u, 3 u)-6 g(2 u, 2 u)+15 g(u, u)\|+\|g(4 u, 4 u)-4 g(3 u, 3 u)+4 g(2 u, 2 u)+4 g(u, u)\| \\
& \quad \leq 4 \alpha(u, u, u, u)+\alpha(2 u, u, 2 u, u)
\end{aligned}
$$

for all $u \in G$. From (75), we arrive

$\|g(4 u, 4 u)-20 g(2 u, 2 u)+64 g(u, u)\| \leq \beta(u)$

where

$\beta(u)=4 \alpha(u, u, u, u)+\alpha(2 u, u, 2 u, u)$

for all $u \in G$. It is easy to see from (76) that

$\|g(4 u, 4 u)-16 g(2 u, 2 u)-4(g(2 u, 2 u)-16 g(u, u))\| \leq \beta(u)$

for all $u \in G$. Using (21) in (78), we obtain

$\left\|q_{2}(2 u, 2 u)-4 q_{2}(u, u)\right\| \leq \beta(u)$

for all $u \in G$. The rest of the proof is similar lines to that of Theorem 3.1.

The following Corollary is an immediate consequence of Theorem 4.1 concerning the Ulam-Hyers, Ulam-HyersRassias, Ulam-Gavruta-Rassias and Ulam-JRassias stabilities of (5).

Corollary 4.2 Let $D g: G^{2} \rightarrow H$ be a mapping and there exits real numbers $\rho$ and s such that

$\|D g(x, y, u, v)\|$

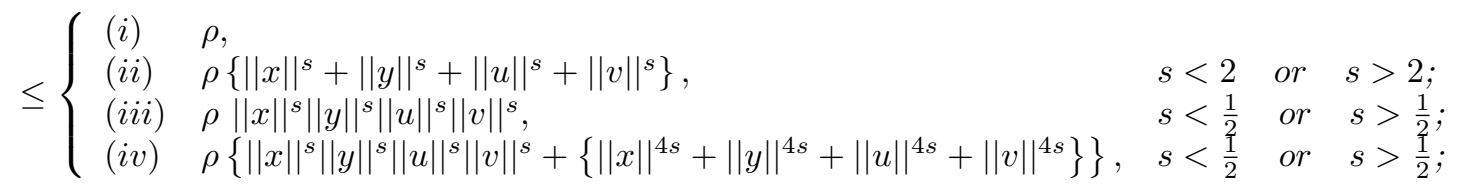

for all $x, y, u, v \in G$, then there exists a unique 2- variable quadratic function $Q_{2}(u, u): G^{2} \rightarrow H$ such that

$$
\left\|g(2 u, 2 u)-8 g(u, u)-Q_{2}(u, u)\right\| \leq \begin{cases}(i) & \frac{5 \rho}{4}, \\ \text { (ii) } & \frac{\left(18+2^{s+1}\right) \rho\|u\|^{s}}{\left|4-2^{s}\right|}, \\ \text { (iii) } & \frac{\left.\left(4+2^{2 s}\right) \rho|| u\right|^{4 s}}{\left|4-2^{4 s}\right|} \\ \text { (iv) } & \left(\frac{22+2^{2 s}+2^{4 s+1}}{\left|4-2^{4 s}\right|}\right) \rho\|u\|^{4 s}\end{cases}
$$

for all $u \in G$.

Theorem 4.3 Let $j= \pm 1$. Let $D g: G^{2} \rightarrow H$ be an even mapping for which there exist a function $\alpha: G^{4} \rightarrow[0, \infty)$ with the condition

$\lim _{n \rightarrow \infty} \frac{1}{16^{n j}} \alpha\left(2^{n j} x, 2^{n j} y, 2^{n j} u, 2^{n j} v\right)=0$

such that the functional inequality

$\|D g(x, y, u, v)\| \leq \alpha(x, y, u, v)$

for all $x, y, u, v \in G$. Then there exists a unique 2-variable quartic mapping $Q_{4}(u, u): G^{2} \rightarrow H$ satisfying the functional equation (5) and

$\left\|g(2 u, 2 u)-4 g(u, u)-Q_{4}(u, u)\right\| \leq \frac{1}{16} \sum_{k=\frac{1-j}{2}}^{\infty} \frac{\beta\left(2^{k j} u\right)}{16^{k j}}$ 
for all $u \in G$, where $\beta\left(2^{k j} u\right)$ and $Q_{4}(u, u)$ are defined by

$\beta\left(2^{k j} u\right)=4 \alpha\left(2^{k j} u, 2^{k j} u, 2^{k j} u, 2^{k j} u\right)+\alpha\left(2^{(k+1) j} u, 2^{k j} u, 2^{(k+1) j} u, 2^{k j} u\right)$

$Q_{4}(u, u)=\lim _{n \rightarrow \infty} \frac{1}{16^{n j}}\left(g\left(2^{(n+1) j} u, 2^{(n+1) j} u\right)-4 g\left(2^{n j} u, 2^{n j} u\right)\right)$

for all $u \in G$.

Proof. It is easy to see from (76) that

$\|g(4 u, 4 u)-4 g(2 u, 2 u)-16(g(2 u, 2 u)-4 g(u, u))\| \leq \beta(u)$

for all $u \in G$. Using (18) in (87), we obtain

$\left\|q_{4}(2 u, 2 u)-16 q_{4}(u, u)\right\| \leq \beta(u)$

for all $u \in G$. The rest of proof is similar tracing to that of Theorem 3.1.

The following Corollary is an immediate consequence of Theorem 4.3 concerning the Ulam-Hyers, Ulam-HyersRassias, Ulam-Gavruta-Rassias and Ulam-JRassias stabilities of (5).

Corollary 4.4 Let $D g: G^{2} \rightarrow H$ be a mapping and there exits real numbers $\rho$ and $s$ such that

$\|D g(x, y, u, v)\|$

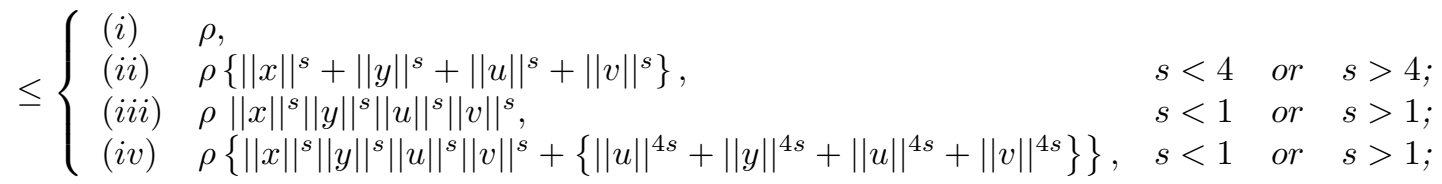

for all $x, y, u, v \in G$, then there exists a unique 2-variable quartic function $Q_{4}(u, u): G^{2} \rightarrow H$ such that

$$
\left\|g(2 u, 2 u)-4 c g(u, u)-Q_{4}(u, u)\right\| \leq \begin{cases}(i) \quad \frac{5 \rho}{15}, \\ (\text { ii }) & \frac{\left(18+2^{s+1}\right) \rho \|\left. u\right|^{s}}{\left|16-2^{s}\right|} \\ (\text { iii }) & \frac{\left(4+2^{2 s}\right) \rho|| u||^{4 s}}{\left|16-2^{4 s}\right|} \\ \text { (iv) } & \left(\frac{22+2^{2 s}+2^{4 s+1}}{\left|16-2^{4 s}\right|}\right) \rho\|u\|^{4 s}\end{cases}
$$

for all $u \in G$.

Theorem 4.5 Let $j= \pm 1$. Let $D g: G^{2} \rightarrow H$ be a mapping for which there exist a function $\alpha: G^{4} \rightarrow[0, \infty)$ with the condition given in (68) and (82) respectively, such that the functional inequality

$\|D g(x, y, u, v)\| \leq \alpha(x, y, u, v)$

for all $x, y, u, v \in G$. Then there exists a unique 2-variable quadratic mapping $Q_{2}(u, u): G^{2} \rightarrow H$ and a unique 2-variable quartic mapping $Q_{4}(u, u): G^{2} \rightarrow H$ satisfying the functional equation (5) and

$\left\|g(u, u)-Q_{2}(u, u)-Q_{4}(u, u)\right\| \leq \frac{1}{12}\left\{\frac{1}{4} \sum_{k=\frac{1-j}{2}}^{\infty} \frac{\beta\left(2^{k j} u\right)}{4^{k j}}+\frac{1}{16} \sum_{k=\frac{1-j}{2}}^{\infty} \frac{\beta\left(2^{k j} u\right)}{16^{k j}}\right\}$

for all $u \in G$, where $\beta\left(2^{k j} u\right), Q_{2}(u, u)$ and $Q_{4}(u, u)$ are respectively defined in (71), (72) and (86) for all $u \in G$.

Proof. By Theorems 4.1 and 4.3, there exists a unique 2-variable quadratic function $Q_{2_{1}}(u, u): G^{2} \rightarrow H$ and a unique 2-variable quartic function $Q_{4_{1}}(u, u): G^{2} \rightarrow H$ such that

$\left\|g(2 u, 2 u)-16 g(u, u)-Q_{2_{1}}(u, u)\right\| \leq \frac{1}{4} \sum_{k=\frac{1-j}{2}}^{\infty} \frac{\beta\left(2^{k j} x\right)}{4^{k j}}$ 
and

$\left\|g(2 u, 2 u)-4 g(u, u)-Q_{4_{1}}(u, u)\right\| \leq \frac{1}{16} \sum_{k=\frac{1-j}{2}}^{\infty} \frac{\beta\left(2^{k j} x\right)}{16^{k j}}$

for all $u \in G$. Now from (93) and (94), one can see that

$$
\begin{aligned}
& \left\|g(u, u)+\frac{1}{12} Q_{2_{1}}(u, u)-\frac{1}{12} Q_{4_{1}}(u, u)\right\| \\
& \quad=\left\|\left\{-\frac{g(2 u, 2 u)}{12}+\frac{16 g(u, u)}{12}+\frac{Q_{2_{1}}(u, u)}{12}\right\}+\left\{\frac{g(2 u, 2 u)}{12}-\frac{4 g(u, u)}{12}-\frac{Q_{4_{1}}(u, u)}{12}\right\}\right\| \\
& \leq \frac{1}{12}\left\{\left\|g(2 u, 2 u)-16 g(u, u)-Q_{2_{1}}(u, u)\right\|+\left\|g(2 u, 2 u)-4 g(u, u)-Q_{4_{1}}(u, u)\right\|\right\} \\
& \quad \leq \frac{1}{12}\left\{\frac{1}{4} \sum_{k=\frac{1-j}{2}}^{\infty} \frac{\beta\left(2^{k j} x\right)}{4^{k j}}+\frac{1}{16} \sum_{k=\frac{1-j}{2}}^{\infty} \frac{\beta\left(2^{k j} x\right)}{16^{k j}}\right\}
\end{aligned}
$$

for all $u \in U$. Thus we obtain (94) by defining $Q_{2}(u, u)=\frac{-1}{12} Q_{2_{1}}(u, u)$ and $Q_{4}(u, u)=\frac{1}{12} Q_{4_{1}}(u, u)$, where $\beta\left(2^{k j} u\right)$, $Q_{2}(u, u)$ and $Q_{4}(u, u)$ are respectively defined in (71), (72) and (86) for all $u \in G$.

The following corollary is the immediate consequence of Theorem 4.5, using Corollaries 4.2 and 4.4 concerning the the Ulam-Hyers, Ulam-Hyers-Rassias, Ulam-Gavruta-Rassias and Ulam-JRassias stabilities of (5).

Corollary 4.6 Let $D g: G^{2} \rightarrow H$ be a mapping and there exits real numbers $\rho$ and $s$ such that

$$
\begin{aligned}
& \|D g(x, y, u, v)\| \\
& \leq\left\{\begin{array}{lll}
(i) & \rho, & s \neq 2,4 ; \\
(i i) & \rho\left\{\|x\|^{s}+\|y\|^{s}+\|u\|^{s}+\|v\|^{s}\right\}, & s \neq \frac{1}{2}, 1 ; \\
(i i i) & \rho\|x\|^{s}\|y\|\left\|^{s}\right\| u\left\|^{s}\right\| v \|^{s}, \\
(i v) & \rho\left\{\|x\|^{s}\|y\|^{s}\|u\|^{s}\|v\|^{s}+\left\{\|x\|^{4 s}+\|y\|^{4 s}+\|u\|^{4 s}+\|v\|^{4 s}\right\}\right\}, & s \neq \frac{1}{2}, 1 ;
\end{array}\right.
\end{aligned}
$$

for all $x, y, u, v \in G$, then there exists a unique 2-variable quadratic mapping $Q_{2}(u, u): G^{2} \rightarrow H$ and a unique 2-variable quartic mapping $Q_{4}(u, u): G^{2} \rightarrow H$ such that

$$
\begin{aligned}
& \left\|g(u, u)-Q_{2}(u, u)-Q_{4}(u, u)\right\| \\
& \quad \leq \begin{cases}(i) & \frac{5 \rho}{12}\left(\frac{1}{3}+\frac{1}{15}\right) \\
(i i) & \frac{\left(18+2^{s+1}\right)}{12}\left(\frac{1}{\left|4-2^{s}\right|}+\frac{1}{\left|16-2^{s}\right|}\right) \rho\|u\|^{s} \\
(i i i) & \frac{\left(4+2^{2 s}\right)}{12}\left(\frac{1}{\mid 4-2^{4 s \mid}}+\frac{1}{\left|16-2^{4 s}\right|}\right) \rho\|u\|^{4 s} \\
(i v) & \frac{\left(22+2^{2 s}+2^{4 s+1}\right)}{12}\left(\frac{1}{\left|4-2^{4 s}\right|}+\frac{1}{\mid 16-2^{4 s \mid}}\right) \rho\|u\|^{4 s}\end{cases}
\end{aligned}
$$

for all $u \in G$.

\section{Stability results: Mixed case}

Theorem 5.1 Let $j= \pm 1$. Let $D g: G^{2} \rightarrow H$ be a mapping for which there exist a function $\alpha: G^{4} \rightarrow[0, \infty)$ with the condition given in (35), (53), (68) and (82) respectively, such that the functional inequality

$\|D g(x, y, u, v)\| \leq \alpha(x, y, u, v)$

for all $x, y, u, v \in G$. Then there exists a unique 2-variable additive mapping $A(u, u): G^{2} \rightarrow H$, anique 2-variable quadratic mapping $Q_{2}(u, u): G^{2} \rightarrow H$, a unique 2-variable cubic mapping $C(u, u): G^{2} \rightarrow H$ and a unique 2-variable 
quartic mapping $Q_{4}(u, u): G^{2} \rightarrow$ Hsatisfying the functional equation (5) and

$$
\begin{aligned}
& \left\|g(u, u)-A(u, u)-Q_{2}(u, u)-C(u, u)-Q_{4}(u, u)\right\| \\
& \leq \frac{1}{12}\left\{\frac{1}{2} \sum_{k=\frac{1-j}{2}}^{\infty}\left(\frac{\beta\left(2^{k j} u\right)}{2^{k j}}+\frac{\beta\left(-2^{k j} u\right)}{2^{k j}}\right)+\frac{1}{8} \sum_{k=\frac{1-j}{2}}^{\infty}\left(\frac{\beta\left(2^{k j} u\right)}{8^{k j}}+\frac{\beta\left(-2^{k j} u\right)}{8^{k j}}\right)\right\} \\
& \quad+\frac{1}{24}\left\{\frac{1}{4} \sum_{k=\frac{1-j}{2}}^{\infty}\left(\frac{\beta\left(2^{k j} u\right)}{4^{k j}}+\frac{\beta\left(-2^{k j} u\right)}{4^{k j}}\right)+\frac{1}{16} \sum_{k=\frac{1-j}{2}}^{\infty}\left(\frac{\beta\left(2^{k j} u\right)}{16^{k j}}+\frac{\beta\left(-2^{k j} u\right)}{16^{k j}}\right)\right\}
\end{aligned}
$$

for all $u \in G$, where $\beta\left(2^{k j} u\right), A(u, u), C(u, u), Q_{2}(u, u)$ and $Q_{4}(u, u)$ are respectively defined in (38), (39), (57), (72) and (86) for all $u \in G$.

Proof. Let $g_{o}(u, u)=\frac{g(u, u)-g(-u,-u)}{2}$ for all $u \in G$. Then $g_{o}(0,0)=0$ and $g_{o}(-u,-u)=-g_{o}(u, u)$ for all $u \in G$. Hence

$$
\left\|D g_{o}(x, y, u, v)\right\| \leq \frac{1}{2}\{\alpha(x, y, u, v)+\alpha(-x,-y,-u,-v)\}
$$

for all $x, y, u, v \in G$. By Theorem 3.5, there exists a unique 2-variable additive function $A(u, u): G^{2} \rightarrow H$ and a unique 2-variable cubic function $C(u, u): G^{2} \rightarrow H$ such that

$$
\begin{aligned}
& \left\|g_{o}(u, u)-A(u, u)-C(u, u)\right\| \\
& \quad \leq \frac{1}{12}\left\{\frac{1}{2} \sum_{k=\frac{1-j}{2}}^{\infty}\left(\frac{\beta\left(2^{k j} u\right)}{2^{k j}}+\frac{\beta\left(-2^{k j} u\right)}{2^{k j}}\right)+\frac{1}{8} \sum_{k=\frac{1-j}{2}}^{\infty}\left(\frac{\beta\left(2^{k j} u\right)}{8^{k j}}+\frac{\beta\left(-2^{k j} u\right)}{8^{k j}}\right)\right\}
\end{aligned}
$$

for all $u \in G$. Also, let $g_{e}(u, u)=\frac{g(u, u)+g(-u,-u)}{2}$ for all $u \in G$. Then $g_{e}(0,0)=0$ and $g_{e}(-u,-u)=g_{e}(u, u)$ for all $u \in G$. Hence

$$
\left\|D g_{e}(x, y, u, v)\right\| \leq \frac{1}{2}\{\alpha(x, y, u, v)+\alpha(-x,-y,-u,-v)\}
$$

for all $x, y, u, v \in G$. By Theorem 4.5, there exists a unique 2-variable quadratic mapping $Q_{2}(u, u): G^{2} \rightarrow H$ and a unique 2-variable quartic mapping $Q_{4}(u, u): G^{2} \rightarrow H$ such that

$$
\begin{aligned}
& \left\|g_{e}(u, u)-Q_{2}(u, u)-Q_{4}(u, u)\right\| \\
& \quad \leq \frac{1}{24}\left\{\frac{1}{4} \sum_{k=\frac{1-j}{2}}^{\infty}\left(\frac{\beta\left(2^{k j} u\right)}{4^{k j}}+\frac{\beta\left(-2^{k j} u\right)}{4^{k j}}\right)+\frac{1}{16} \sum_{k=\frac{1-j}{2}}^{\infty}\left(\frac{\beta\left(2^{k j} u\right)}{16^{k j}}+\frac{\beta\left(-2^{k j} u\right)}{16^{k j}}\right)\right\}
\end{aligned}
$$

for all $u \in G$. Define

$g(u, u)=g_{o}(u, u)+g_{e}(u, u)$

for all $u \in G$. Now from (103), (102) and (100)

$$
\begin{aligned}
& \left\|g(u, u)-A(u, u)-Q_{2}(u, u)-C(u, u)-Q_{4}(u, u)\right\| \\
& \quad=\left\|g_{o}(u, u)+g_{e}(u, u)-A(u, u)-Q_{2}(u, u)-C(u, u)-Q_{4}(u, u)\right\| \\
& \quad \leq\left\|g_{o}(u, u)-A(u, u)-C(u, u)\right\|+\left\|g_{e}(u, u)-Q_{2}(u, u)-Q_{4}(u, u)\right\| \\
& \quad \leq \frac{1}{12}\left\{\frac{1}{2} \sum_{k=\frac{1-j}{2}}^{\infty}\left(\frac{\beta\left(2^{k j} u\right)}{2^{k j}}+\frac{\beta\left(-2^{k j} u\right)}{2^{k j}}\right)+\frac{1}{8} \sum_{k=\frac{1-j}{2}}^{\infty}\left(\frac{\beta\left(2^{k j} u\right)}{8^{k j}}+\frac{\beta\left(-2^{k j} u\right)}{8^{k j}}\right)\right\} \\
& \quad+\frac{1}{24}\left\{\frac{1}{4} \sum_{k=\frac{1-j}{2}}^{\infty}\left(\frac{\beta\left(2^{k j} u\right)}{4^{k j}}+\frac{\beta\left(-2^{k j} u\right)}{4^{k j}}\right)+\frac{1}{16} \sum_{k=\frac{1-j}{2}}^{\infty}\left(\frac{\beta\left(2^{k j} u\right)}{16^{k j}}+\frac{\beta\left(-2^{k j} u\right)}{16^{k j}}\right)\right\}
\end{aligned}
$$


for all $u \in G$, where $\beta\left(2^{k j} u\right), A(u, u), C(u, u), Q_{2}(u, u)$ and $Q_{4}(u, u)$ are respectively defined in (38), (39), (57), (72) and (86) for all $u \in G$.

The following corollary is the immediate consequence of Theorem 5.1, using Corollaries 3.6 and 4.6 concerning the Ulam-Hyers, Ulam-Hyers-Rassias, Ulam-Gavruta-Rassias and Ulam-JRassias stabilities of (5).

Corollary 5.2 Let $D g: G^{2} \rightarrow H$ be a mapping and there exits real numbers $\rho$ and $s$ such that

$$
\begin{aligned}
& \|D g(x, y, u, v)\| \\
& \leq\left\{\begin{array}{lll}
(i) & \rho, \\
(i i) & \rho\left\{\|x\|^{s}+\|y\|^{s}+\|u\|^{s}+\|v\|^{s}\right\}, & s \neq 1,2,3,4 \\
(i i i) & \rho\|x\|\left\|^{s}\right\| y\|\|^{s}\|u\|^{s}\|v\|^{s}, & s \neq \frac{1}{4}, \frac{1}{2}, \frac{3}{4}, 1 \\
(i v) & \rho\left\{\|x\|^{s}\|y\|^{s}\|u\|^{s}\|v\|^{s}+\left\{\|x\|^{4 s}+\|y\|^{4 s}+\|u\|^{4 s}+\|v\|^{4 s}\right\}\right\}, & s \neq \frac{1}{4}, \frac{1}{2}, \frac{3}{4}, 1
\end{array}\right.
\end{aligned}
$$

for all $x, y, u, v \in G$, then there exists a unique 2-variable additive mapping $A(u, u): G^{2} \rightarrow H$, a unique 2-variable quadratic mapping $Q_{2}(u, u): G^{2} \rightarrow H$, a unique 2-variable cubic mapping $C(u, u): G^{2} \rightarrow H$ and a unique 2-variable quartic mapping $Q_{4}(u, u): G^{2} \rightarrow H$ such that

$$
\begin{aligned}
& \left\|g(u, u)-A(u, u)-Q_{2}(u, u)-C(u, u)-Q_{4}(u, u)\right\| \\
& \quad \leq \begin{cases}(i) & \frac{5 \rho}{6}\left(1+\frac{1}{7}+\frac{1}{2 \cdot 3}+\frac{1}{2 \cdot 15}\right), \\
(i i) & \frac{\left(18+2^{s+1}\right)}{6}\left(\frac{1}{\left|2-2^{s}\right|}+\frac{1}{\left|8-2^{s}\right|}+\frac{1}{2\left|4-2^{s}\right|}+\frac{1}{2\left|16-2^{s}\right|}\right) \rho\|u\|^{s}, \\
\text { (iii) } & \frac{\left(4+2^{2 s}\right)}{6}\left(\frac{1}{\mid 2-2^{4 s \mid}}+\frac{1}{\mid 8-2^{4 s \mid}}+\frac{1}{2 \mid 4-2^{4 s \mid}}+\frac{1}{2 \mid 16-2^{4 s \mid}}\right) \rho \|\left. u\right|^{4 s} \\
\text { (iv) } & \frac{\left(22+2^{2 s}+2^{4 s+1}\right)}{6}\left(\frac{1}{\mid 2-2^{4 s \mid}}+\frac{1}{\mid 8-2^{4 s \mid}}+\frac{1}{2 \mid 4-2^{4 s \mid}}+\frac{1}{2 \mid 16-2^{4 s \mid}}\right) \rho\|u\|^{4 s}\end{cases}
\end{aligned}
$$

for all $u \in G$.

\section{Counter examples for non stable cases: Odd case}

Now the author provide an example to illustrate that the functional equation (5) is not stable for $s=1$ in condition (ii) of Corollary 3.2.

Example 6.1 Let $\alpha: R^{4} \rightarrow R$ be a function defined by

$$
\alpha(u)= \begin{cases}\mu u, & \text { if }|u|<1 \\ \mu, & \text { otherwise }\end{cases}
$$

where $\mu>0$ is a constant, and define a function $g: R^{2} \rightarrow R$ by

$$
g(u, u)=\sum_{n=0}^{\infty} \frac{\alpha\left(2^{n} u\right)}{2^{n}} \quad \text { for all } \quad u \in R .
$$

Then $g$ satisfies the functional inequality

$|D g(x, y, u, v)| \leq 52 \mu(|x|+|y|+|u|+|v|)$

for all $x, y, u, v \in R$. Then there do not exist a additive mapping $A: R^{2} \rightarrow R$ and a constant $\delta>0$ such that

$|g(2 u, 2 u)-8 g(u, u)-A(u, u)| \leq \delta|u| \quad$ for all $\quad u \in R$.

Proof. Now

$$
|g(x, x)| \leq \sum_{n=0}^{\infty} \frac{\left|\alpha\left(2^{n} u\right)\right|}{\left|2^{n}\right|}=\sum_{n=0}^{\infty} \frac{\mu}{2^{n}}=2 \mu .
$$

Therefore we see that $g$ is bounded. We are going to prove that $g$ satisfies (107). 
If $x=y=u=v=0$ then (107) is trivial. If $|x|+|y|+|u|+|v| \geq \frac{1}{2}$ then the left hand side of (107) is less than $52 \mu$. Now suppose that $0<|x|+|y|+|u|+|v|<\frac{1}{2}$. Then there exists a positive integer $k$ such that

$\frac{1}{2^{k}} \leq|x|+|y|+|u|+|v|<\frac{1}{2^{k-1}}$

so that $2^{k-1} x<\frac{1}{2}, 2^{k-1} y<\frac{1}{2}, 2^{k-1} u<\frac{1}{2}, 2^{k-1} v<\frac{1}{2}$ and consequently

$2^{k-1}(y, v), 2^{k-1}(-y,-v), 2^{k-1}(2 y, 2 v), 2^{k-1}(-2 y,-2 v), 2^{k-1}(x, u), 2^{k-1}(x+y, u+v)$,
$2^{k-1}(x-y, u-v), 2^{k-1}(x+2 y, u+2 v), 2^{k-1}(x-2 y, u-2 v) \in(-1,1)$.

Therefore for each $n=0,1, \ldots, k-1$, we have

$2^{n}(y, v), 2^{n}(-y,-v), 2^{n}(2 y, 2 v), 2^{n}(-2 y,-2 v), 2^{n}(x, u), 2^{n}(x+y, u+v)$,

$2^{n}(x-y, u-v), 2^{n}(x+2 y, u+2 v), 2^{n}(x-2 y, u-2 v) \in(-1,1)$.

and

$$
\begin{aligned}
& \alpha\left(2^{n}(x+2 y, u+2 v)\right)+\alpha\left(2^{n}(x-2 y, u-2 v)\right)-4 \alpha\left(2^{n}(x+y, u+v)\right) \\
& -4 \alpha\left(2^{n}(x-y, u-v)\right)+6 \alpha\left(2^{n}(x, u)\right)-\alpha\left(2^{n}(2 y, 2 v)\right)-\alpha\left(2^{n}(-2 y,-2 v)\right) \\
& +4 \alpha\left(2^{n}(y, v)\right)+4 \alpha\left(2^{n}(-y,-v)\right)=0
\end{aligned}
$$

for $n=0,1, \ldots, k-1$. From the definition of $f$ and (109), we obtain that

$$
\begin{aligned}
& \mid g(x+2 y, u+2 v)+g(x-2 y, u-2 v)-4[g(x+y, u+v)+g(x-y, u-v)] \\
& \quad+6 g(x, u)-g(2 y, 2 v)-g(-2 y,-2 v)+4 g(y, v)+4 g(-y,-v) \mid \\
& \leq \sum_{n=0}^{\infty} \frac{1}{2^{n}} \mid \alpha\left(2^{n}(x+2 y, u+2 v)\right)+\alpha\left(2^{n}(x-2 y, u-2 v)\right)-4 \alpha\left(2^{n}(x+y, u+v)\right) \\
& \quad-4 \alpha\left(2^{n}(x-y, u-v)\right)-6 \alpha\left(2^{n}(x, u)\right)-\alpha\left(2^{n}(2 y, 2 v)\right)-\alpha\left(2^{n}(-2 y,-2 v)\right) \\
& \quad+4 \alpha\left(2^{n}(y, v)\right)+4 \alpha\left(2^{n}(-y,-v)\right) \mid \\
& \leq \sum_{n=k}^{\infty} \frac{1}{2^{n}} \mid \alpha\left(2^{n}(x+2 y, u+2 v)\right)+\alpha\left(2^{n}(x-2 y, u-2 v)\right)-4 \alpha\left(2^{n}(x+y, u+v)\right) \\
& \quad-4 \alpha\left(2^{n}(x-y, u-v)\right)-6 \alpha\left(2^{n}(x, u)\right)-\alpha\left(2^{n}(2 y, 2 v)\right)-\alpha\left(2^{n}(-2 y,-2 v)\right) \\
& \quad+4 \alpha\left(2^{n}(y, v)\right)+4 \alpha\left(2^{n}(-y,-v)\right) \mid \\
& \leq \sum_{n=k}^{\infty} \frac{1}{2^{n}} 26 \mu=26 \mu \times \frac{2}{2^{k}}=52 \mu(|x|+|y|+|u|+|v|) .
\end{aligned}
$$

Thus $g$ satisfies (107) for all $x, y, u, v \in R$ with $0<|x|+|y|+|u|+|v|<\frac{1}{2}$.

We claim that the additive functional equation (5) is not stable for $s=1$ in condition ( $i i$ ) Corollary 3.2. Suppose on the contrary that there exist a additive mapping $A: R^{2} \rightarrow R$ and a constant $\delta>0$ satisfying (108). Since $g$ is bounded and continuous for all $u \in R, A$ is bounded on any open interval containing the origin and continuous at the origin. In view of Theorem 3.1, $A$ must have the form $A(u, u)=c u$ for any $u$ in $R$. Thus we obtain that

$|g(2 u, 2 u)-8 g(u, u)-A(u, u)| \leq(\delta+|c|)|u|$.

But we can choose a positive integer $m$ with $m \mu>\delta+|c|$.

If $u \in\left(0, \frac{1}{2^{m-1}}\right)$, then $2^{n} u \in(0,1)$ for all $n=0,1, \ldots, m-1$. For this $u$, we get

$$
g(2 u, 2 u)-8 g(u, u)=\sum_{n=0}^{\infty} \frac{\alpha\left(2^{n} u\right)}{2^{n}} \geq \sum_{n=0}^{m-1} \frac{\mu\left(2^{n} u\right)}{2^{n}}=m \mu u>(\beta+|c|) u
$$


which contradicts (110). Therefore the additive functional equation (5) is not stable in sense of Ulam, Hyers and Rassias if $s=1$, assumed in the inequality condition (ii) of (51).

A counter example to illustrate the non stability in condition ( $i i i)$ of Corollary 3.2 is given in the following example.

Example 6.2 Let $s$ be such that $0<s<\frac{1}{4}$. Then there is a function $g: R^{2} \rightarrow R$ and a constant $\lambda>0$ satisfying

$|D g(x, y, u, v)| \leq \lambda|x|^{\frac{s}{4}}|y|^{\frac{s}{4}}|u|^{\frac{s}{4}}|v|^{\frac{1-3 s}{4}}$

for all $x, y, u, v \in R$ and

$\sup _{u \neq 0} \frac{|g(2 u, 2 u)-8 g(u, u)-A(u, u)|}{|u|}=+\infty$

for every additive mapping $A(u, u): R^{2} \rightarrow R$.

Proof. If we take

$g(u, u)= \begin{cases}(u, u) \ln |u, u| & \text { if } u \neq 0, \\ 0, & \text { if } u=0 .\end{cases}$

Then from the relation (112), it follows that

$$
\begin{aligned}
\sup _{u \neq 0} \frac{|g(2 u, 2 u)-8 g(u, u)-A(u, u)|}{|u|} & \geq \sup _{\substack{n \in N \\
n \neq 0}} \frac{|g(2 n, 2 n)-8 g(n, n)-A(n, n)|}{|n|} \\
& =\sup _{\substack{n \in N \\
n \neq 0}} \frac{|n(2,2) \ln | 2 n, 2 n|-8 n(1,1) \ln | n, n|-n A(1,1)|}{|n|} \\
& =\sup _{\substack{n \in N \\
n \neq 0}}|(2,2) \ln | 2 n, 2 n|-8(1,1) \ln | n, n|-A(1,1)|=\infty .
\end{aligned}
$$

We have to prove (111) is true.

Case (i): If $x, y, u, v>0$ in (111) then,

$$
\begin{aligned}
& \mid g(x+2 y, u+2 v)+g(x-2 y, u-2 v)-4[g(x+y, u+v)+g(x-y, u-v)] \\
& \quad+6 g(x, u)-g(2 y, 2 v)-g(-2 y,-2 v)+4 g(y, v)+4 g(-y,-v) \mid \\
& =|(x+2 y, u+2 v) \ln | x+2 y, u+2 v|+(x-2 y, u-2 v) \ln | x-2 y, u-2 v \mid \\
& \quad-4[(x+y, u+v) \ln |x+y, u+v|+(x-y, u-v) \ln |x-y, u-v|]+6(x, u) \ln |x, u| \\
& \quad-(2 y, 2 v) \ln |2 y, 2 v|-(-2 y,-2 v) \ln |-2 y,-2 v| \\
& \quad+4[(y, v) \ln |y, v|+(-y,-v) \ln |-y,-v|] \mid .
\end{aligned}
$$

Set $x=v_{1}, y=v_{2}, u=v_{3}, v=v_{4}$ it follows that

$$
\begin{aligned}
& \mid g(x+2 y, u+2 v)+g(x-2 y, u-2 v)-4[g(x+y, u+v)+g(x-y, u-v)] \\
& \quad+6 g(x, u)-g(2 y, 2 v)-g(-2 y,-2 v)+4 g(y, v)+4 g(-y,-v) \mid \\
& =\left|\left(v_{1}+2 v_{2}, v_{3}+2 v_{4}\right) \ln \right| v_{1}+2 v_{2}, v_{3}+2 v_{4}\left|+\left(v_{1}-2 v_{2}, v_{3}-2 v_{4}\right) \ln \right| v_{1}-2 v_{2}, v_{3}-2 v_{4} \mid \\
& \quad-4\left[\left(v_{1}+v_{2}, v_{3}+v_{4}\right) \ln \left|v_{1}+v_{2}, v_{3}+v_{4}\right|+\left(v_{1}-v_{2}, v_{3}-v_{4}\right) \ln \left|v_{1}-v_{2}, v_{3}-v_{4}\right|\right] \\
& \quad \quad+6\left(v_{1}, v_{3}\right) \ln \left|v_{1}, v_{3}\right|-\left(2 v_{2}, 2 v_{4}\right) \ln \left|2 v_{2}, 2 v_{4}\right|-\left(-2 v_{2},-2 v_{4}\right) \ln \left|-2 v_{2},-2 v_{4}\right| \\
& \quad \quad+4\left[\left(v_{2}, v_{4}\right) \ln \left|v_{2}, v_{4}\right|+\left(-v_{2},-v_{4}\right) \ln \left|-v_{2},-v_{4}\right|\right] \mid . \\
& =\mid g\left(v_{1}+2 v_{2}, v_{3}+2 v_{4}\right)+g\left(v_{1}-2 v_{2}, v_{3}-2 v_{4}\right)-4\left[g\left(v_{1}+v_{2}, v_{3}+v_{4}\right)+g\left(v_{1}-v_{2}, v_{3}-v_{4}\right)\right] \\
& \quad+6 g\left(v_{1}, v_{3}\right)-g\left(2 v_{2}, 2 v_{4}\right)-g\left(-2 v_{2},-2 v_{4}\right)+4 g\left(v_{2}, v_{4}\right)+4 g\left(-v_{2},-v_{4}\right) \mid \\
& \leq \lambda\left|v_{1}\right|^{\frac{s}{4}}\left|v_{2}\right|^{\frac{s}{4}}\left|v_{3}\right|^{\frac{s}{4}}\left|v_{4}\right|^{\frac{1-3 s}{4}} \\
& =\lambda|x|^{\frac{s}{4}}|y|^{\frac{s}{4}}|u|^{\frac{s}{4}}|v|^{\frac{1-3 s}{4}} .
\end{aligned}
$$


For the cases (ii) $x, y, u, v<0$, (iii) $x, z>0, u, v<0$, (iv) $x, z>0, u, v<0$ and $(v) \quad x=y=u=v=0$, the proof is similar tracing to that of Case $(i)$.

Now we will provide an example to illustrate that the functional equation (5) is not stable for $s=\frac{1}{4}$ in condition (iv) of Corollary 3.2.

Example 6.3 Let $\alpha: R \rightarrow R$ be a function defined by

$$
\alpha(u)= \begin{cases}\mu u, & \text { if }|u|<\frac{1}{4} \\ \frac{\mu}{4}, & \text { otherwise }\end{cases}
$$

where $\mu>0$ is a constant, and define a function $g: R^{2} \rightarrow R$ by

$$
g(u, u)=\sum_{n=0}^{\infty} \frac{\alpha\left(2^{n} u\right)}{2^{n}} \quad \text { for all } \quad u \in R .
$$

Then $g$ satisfies the functional inequality

$|D g(x, y, u, v)| \leq 13 \mu\left(|x|^{\frac{1}{4}}|y|^{\frac{1}{4}}|u|^{\frac{1}{4}}|v|^{\frac{1}{4}}+\{|x|+|y|+|u|+|v|\}\right)$

for all $x, y, u, v \in R$. Then there do not exist a additive mapping $A: R^{2} \rightarrow R$ and a constant $\delta>0$ such that

$|g(2 u, 2 u)-8 g(u, u)-A(u, u)| \leq \delta|u| \quad$ for all $\quad u \in R$.

Proof. Now

$$
|g(u, u)| \leq \sum_{n=0}^{\infty} \frac{\left|\alpha\left(2^{n} u\right)\right|}{\left|2^{n}\right|}=\sum_{n=0}^{\infty} \frac{1}{2^{n}} \times \frac{\mu}{4}=\frac{\mu}{2} .
$$

Therefore we see that $g$ is bounded. We are going to prove that $g$ satisfies (113).

If $x=y=u=v=0$ then (113) is trivial.

If $|x|^{\frac{1}{4}}|y|^{\frac{1}{4}}|u|^{\frac{1}{4}}|v|^{\frac{1}{4}}+\{|x|+|y|+|u|+|v|\} \geq \frac{1}{2}$ then the left hand side of (113) is less than $13 \mu$. The rest of the proof is similar tracing to that of Example 6.1.

Now the author provide an example to illustrate that the functional equation (5) is not stable for $s=3$ in condition (ii) of Corollary 3.4.

Example 6.4 Let $\alpha: R^{4} \rightarrow R$ be a function defined by

$$
\alpha(u)= \begin{cases}\mu u^{3}, & \text { if }|u|<1 \\ \mu, & \text { otherwise }\end{cases}
$$

where $\mu>0$ is a constant, and define a function $g: R^{2} \rightarrow R$ by

$$
g(u, u)=\sum_{n=0}^{\infty} \frac{\alpha\left(2^{n} u\right)}{8^{n}} \quad \text { for all } \quad u \in R .
$$

Then $g$ satisfies the functional inequality

$|D g(x, y, u, v)| \leq \frac{52 \times 8^{3}}{7} \mu\left(|x|^{3}+|y|^{3}+|u|^{3}+|v|^{3}\right)$

for all $x, y, u, v \in R$. Then there do not exist a cubic mapping $C: R^{2} \rightarrow R$ and a constant $\delta>0$ such that

$|g(2 u, 2 u)-2 g(u, u)-C(u, u)| \leq \delta|u|^{3} \quad$ for all $u \in R$.

Proof. Now

$$
|g(x, x)| \leq \sum_{n=0}^{\infty} \frac{\left|\alpha\left(2^{n} u\right)\right|}{\left|8^{n}\right|}=\sum_{n=0}^{\infty} \frac{\mu}{8^{n}}=\frac{8}{7} \mu .
$$

Therefore we see that $g$ is bounded. We are going to prove that $g$ satisfies (115). 
If $x=y=u=v=0$ then (115) is trivial. If $|x|+|y|+|u|+|v| \geq \frac{1}{8}$ then the left hand side of (115) is less than $\frac{52 \times 8}{7} \mu$. The rest of the proof is similar tracing to that of Example 6.1.

A counter example to illustrate the non stability in condition (iii) of Corollary 3.4 is given in the following example.

Example 6.5 Let $s$ be such that $0<s<\frac{3}{4}$. Then there is a function $g: R^{2} \rightarrow R$ and a constant $\lambda>0$ satisfying

$|D g(x, y, u, v)| \leq \lambda|x|^{\frac{s}{4}}|y|^{\frac{s}{4}}|u|^{\frac{s}{4}}|v|^{\frac{3-3 s}{4}}$

for all $x, y, u, v \in R$ and

$\sup _{u \neq 0} \frac{|g(2 u, 2 u)-2 g(u, u)-C(u, u)|}{|u|^{3}}=+\infty$

for every cubic mapping $C(u, u): R^{2} \rightarrow R$.

Proof. If we take

$g(u, u)= \begin{cases}(u, u)^{3} \ln |u, u| & \text { if } u \neq 0, \\ 0, & \text { if } u=0 .\end{cases}$

The rest of the proof is similar tracing to that of Example 6.2.

Now we will provide an example to illustrate that the functional equation (5) is not stable for $s=\frac{3}{4}$ in condition (iv) of Corollary 3.4.

Example 6.6 Let $\alpha: R \rightarrow R$ be a function defined by

$$
\alpha(u)= \begin{cases}\mu u^{3}, & \text { if }|u|<\frac{3}{4} \\ \frac{3 \mu}{4}, & \text { otherwise }\end{cases}
$$

where $\mu>0$ is a constant, and define a function $g: R^{2} \rightarrow R$ by

$$
g(u, u)=\sum_{n=0}^{\infty} \frac{\alpha\left(2^{n} u\right)}{8^{n}} \quad \text { for all } \quad u \in R .
$$

Then $g$ satisfies the functional inequality

$|D g(x, y, u, v)| \leq \frac{156 \times 8^{2} \mu}{7}\left(|x|^{\frac{3}{4}}|y|^{\frac{3}{4}}|u|^{\frac{3}{4}}|v|^{\frac{3}{4}}+\left\{|x|^{3}+|y|^{3}+|u|^{3}+|v|^{3}\right\}\right)$

for all $x, y, u, v \in R$. Then there do not exist a cubic mapping $C: R^{2} \rightarrow R$ and a constant $\delta>0$ such that

$|g(2 u, 2 u)-2 g(u, u)-C(u, u)| \leq \delta|u|^{3} \quad$ for all $\quad u \in R$.

Proof. Now

$$
|g(u, u)| \leq \sum_{n=0}^{\infty} \frac{\left|\alpha\left(2^{n} u\right)\right|}{\left|2^{n}\right|}=\sum_{n=0}^{\infty} \frac{1}{8^{n}} \times \frac{3 \mu}{4}=\frac{6 \mu}{7} .
$$

Therefore we see that $g$ is bounded. We are going to prove that $g$ satisfies (113).

If $x=y=u=v=0$ then (113) is trivial.

If $|x|^{\frac{3}{4}}|y|^{\frac{3}{4}}|u|^{\frac{3}{4}}|v|^{\frac{3}{4}}+\left\{|x|^{3}+|y|^{3}+|u|^{3}+|v|^{3}\right\} \geq \frac{1}{8}$ then the left hand side of (113) is less than $\frac{156 \mu}{7}$. The rest of the proof is similar tracing to that of Example 6.1. 


\section{Counter examples for non stable cases: Even case}

Now the author provide an example to illustrate that the functional equation (5) is not stable for $s=2$ in condition (ii) of Corollary 4.2 .

Example 7.1 Let $\alpha: R^{4} \rightarrow R$ be a function defined by

$$
\alpha(u)= \begin{cases}\mu u^{2}, & \text { if }|u|<1 \\ \mu, & \text { otherwise }\end{cases}
$$

where $\mu>0$ is a constant, and define a function $g: R^{2} \rightarrow R$ by

$$
g(u, u)=\sum_{n=0}^{\infty} \frac{\alpha\left(2^{n} u\right)}{4^{n}} \quad \text { for all } \quad u \in R .
$$

Then $g$ satisfies the functional inequality

$|D g(x, y, u, v)| \leq \frac{26 \times 4}{3} \mu\left(|x|^{2}+|y|^{2}+|u|^{2}+|v|^{2}\right)$

for all $x, y, u, v \in R$. Then there do not exist a quadratic mapping $Q_{2}: R^{2} \rightarrow R$ and a constant $\delta>0$ such that

$\left|g(2 u, 2 u)-16 g(u, u)-Q_{2}(u, u)\right| \leq \delta|u|^{2} \quad$ for all $\quad u \in R$.

Proof. Now

$$
|g(u, u)| \leq \sum_{n=0}^{\infty} \frac{\left|\alpha\left(2^{n} u\right)\right|}{\left|4^{n}\right|}=\sum_{n=0}^{\infty} \frac{\mu}{4^{n}}=\frac{4}{3} \mu .
$$

Therefore we see that $g$ is bounded. We are going to prove that $g$ satisfies (121).

If $x=y=u=v=0$ then (121) is trivial. If $|x|+|y|+|u|+|v| \geq \frac{1}{4}$ then the left hand side of (121) is less than $\frac{26 \times 4}{3} \mu$. The rest of the proof is similar tracing to that of Example 6.1.

A counter example to illustrate the non stability in condition (iii) of Corollary 4.2 is given in the following example.

Example 7.2 Let $s$ be such that $0<s<\frac{1}{2}$. Then there is a function $g: R^{2} \rightarrow R$ and a constant $\lambda>0$ satisfying

$|D g(x, y, u, v)| \leq \lambda|x|^{\frac{s}{2}}|y|^{\frac{s}{2}}|u|^{\frac{s}{2}}|v|^{\frac{1-3 s}{2}}$

for all $x, y, u, v \in R$ and

$\sup _{u \neq 0} \frac{\left|g(2 u, 2 u)-16 g(u, u)-Q_{2}(u, u)\right|}{|u|^{2}}=+\infty$

for every quadratic mapping $Q_{2}(u, u): R^{2} \rightarrow R$.

Proof. If we take

$g(u, u)= \begin{cases}(u, u)^{2} \ln |u, u| & \text { if } u \neq 0, \\ 0, & \text { if } u=0 .\end{cases}$

The rest of the proof is similar tracing to that of Example 6.2.

Now we will provide an example to illustrate that the functional equation (5) is not stable for $s=\frac{1}{2}$ in condition (iv) of Corollary 4.2.

Example 7.3 Let $\alpha: R \rightarrow R$ be a function defined by

$$
\alpha(u)= \begin{cases}\mu u^{2}, & \text { if }|u|<\frac{1}{2} \\ \frac{\mu}{2}, & \text { otherwise }\end{cases}
$$


where $\mu>0$ is a constant, and define a function $g: R^{2} \rightarrow R$ by

$$
g(u, u)=\sum_{n=0}^{\infty} \frac{\alpha\left(2^{n} u\right)}{4^{n}} \quad \text { for all } \quad u \in R .
$$

Then $g$ satisfies the functional inequality

$|D g(x, y, u, v)| \leq \frac{26 \times 4 \mu}{6}\left(|x|^{\frac{1}{2}}|y|^{\frac{1}{2}}|u|^{\frac{1}{2}}|v|^{\frac{1}{2}}+\left\{|x|^{2}+|y|^{2}+|u|^{2}+|v|^{2}\right\}\right)$

for all $x, y, u, v \in R$. Then there do not exist a quadratic mapping $Q_{2}: R^{2} \rightarrow R$ and a constant $\delta>0$ such that

$\left|g(2 u, 2 u)-16 g(u, u)-Q_{2}(u, u)\right| \leq \delta|u| \quad$ for all $u \in R$.

Proof. Now

$$
|g(u, u)| \leq \sum_{n=0}^{\infty} \frac{\left|\alpha\left(2^{n} u\right)\right|}{\left|4^{n}\right|}=\sum_{n=0}^{\infty} \frac{1}{4^{n}} \times \frac{1 \mu}{2}=\frac{4 \mu}{6} .
$$

Therefore we see that $g$ is bounded. We are going to prove that $g$ satisfies (113).

If $x=y=u=v=0$ then (113) is trivial.

If $|x|^{\frac{1}{2}}|y|^{\frac{1}{2}}|u|^{\frac{1}{2}}|v|^{\frac{1}{2}}+\left\{|x|^{2}+|y|^{2}+|u|^{2}+|v|^{2}\right\} \geq \frac{1}{4}$ then the left hand side of (113) is less than $\frac{26 \times 4 \mu}{6}$. The rest of the proof is similar tracing to that of Example 6.1.

Now the author provide an example to illustrate that the functional equation (5) is not stable for $s=4$ in condition (ii) of Corollary 4.4.

Example 7.4 Let $\alpha: R^{4} \rightarrow R$ be a function defined by

$$
\alpha(u)= \begin{cases}\mu u^{4}, & \text { if }|u|<1 \\ \mu, & \text { otherwise }\end{cases}
$$

where $\mu>0$ is a constant, and define a function $g: R^{2} \rightarrow R$ by

$$
g(u, u)=\sum_{n=0}^{\infty} \frac{\alpha\left(2^{n} u\right)}{16^{n}} \quad \text { for all } \quad u \in R .
$$

Then $g$ satisfies the functional inequality

$|D g(x, y, u, v)| \leq \frac{26 \times 16 \mu}{15} \mu\left(|x|^{4}+|y|^{4}+|u|^{4}+|v|^{4}\right)$

for all $x, y, u, v \in R$. Then there do not exist a quartic mapping $Q_{4}: R^{2} \rightarrow R$ and a constant $\delta>0$ such that

$\left|g(2 u, 2 u)-4 g(u, u)-Q_{4}(u, u)\right| \leq \delta|u|^{4} \quad$ for all $\quad u \in R$.

Proof. Now

$$
|g(u, u)| \leq \sum_{n=0}^{\infty} \frac{\left|\alpha\left(2^{n} u\right)\right|}{\left|16^{n}\right|}=\sum_{n=0}^{\infty} \frac{\mu}{16^{n}}=\frac{16}{15} \mu .
$$

Therefore we see that $g$ is bounded. We are going to prove that $g$ satisfies (127).

If $x=y=u=v=0$ then (127) is trivial. If $|x|+|y|+|u|+|v| \geq \frac{1}{16}$ then the left hand side of (127) is less than $\frac{26 \times 16}{15} \mu$. The rest of the proof is similar tracing to that of Example 6.1. A counter example to illustrate the non stability in condition (iii) of Corollary 4.4 is given in the following example.

Example 7.5 Let $s$ be such that $0<s<1$. Then there is a function $g: R^{2} \rightarrow R$ and a constant $\lambda>0$ satisfying

$|D g(x, y, u, v)| \leq \lambda|x|^{s}|y|^{s}|u|^{s}|v|^{1-3 s}$

for all $x, y, u, v \in R$ and

$\sup _{u \neq 0} \frac{\left|g(2 u, 2 u)-4 g(u, u)-Q_{4}(u, u)\right|}{|u|^{4}}=+\infty$

for every quartic mapping $Q_{u}(u, u): R^{2} \rightarrow R$. 
Proof. If we take

$g(u, u)= \begin{cases}(u, u)^{4} \ln |u, u| & \text { if } u \neq 0, \\ 0, & \text { if } u=0 .\end{cases}$

The rest of the proof is similar tracing to that of Example 6.2.

Now we will provide an example to illustrate that the functional equation (5) is not stable for $s=1$ in condition (iv) of Corollary 4.4.

Example 7.6 Let $\alpha: R \rightarrow R$ be a function defined by

$$
\alpha(u)= \begin{cases}\mu u^{4}, & \text { if }|u|<1 \\ \mu, & \text { otherwise }\end{cases}
$$

where $\mu>0$ is a constant, and define a function $g: R^{2} \rightarrow R$ by

$$
g(u, u)=\sum_{n=0}^{\infty} \frac{\alpha\left(2^{n} u\right)}{16^{n}} \quad \text { for all } \quad u \in R .
$$

Then $g$ satisfies the functional inequality

$|D g(x, y, u, v)| \leq \frac{26 \times 16 \mu}{15}\left(|x|^{\frac{1}{4}}|y|^{\frac{1}{4}}|u|^{\frac{1}{4}}|v|^{\frac{1}{4}}+\{|x|+|y|+|u|+|v|\}\right)$

for all $x, y, u, v \in R$. Then there do not exist a quartic mapping $Q_{4}: R^{2} \rightarrow R$ and a constant $\delta>0$ such that

$\left|g(2 u, 2 u)-4 g(u, u)-Q_{4}(u, u)\right| \leq \delta|u| \quad$ for all $\quad u \in R$.

Proof. Now

$$
|g(u, u)| \leq \sum_{n=0}^{\infty} \frac{\left|\alpha\left(2^{n} u\right)\right|}{\left|16^{n}\right|}=\sum_{n=0}^{\infty} \frac{1}{16^{n}} \mu=\frac{16 \mu}{15} .
$$

Therefore we see that $g$ is bounded. We are going to prove that $g$ satisfies (113).

If $x=y=u=v=0$ then (113) is trivial.

If $|x|^{\frac{1}{4}}|y|^{\frac{1}{4}}|u|^{\frac{1}{4}}|v|^{\frac{1}{4}}+\{|x|+|y|+|u|+|v|\} \geq \frac{1}{4}$ then the left hand side of (131) is less than $\frac{26 \times 16 \mu}{15}$. The rest of the proof is similar tracing to that of Example 6.1.

\section{References}

[1] J. Aczel and J. Dhombres, Functional Equations in Several Variables, Cambridge Univ, Press, 1989.

[2] T. Aoki, On the stability of the linear transformation in Banach spaces, J. Math. Soc. Japan, 2 (1950), 64-66.

[3] M. Arunkumar, Matina J. Rassias, Yanhui Zhang, Ulam - Hyers stability of a 2- variable AC - mixed type functional equation: direct and fixed point methods, Journal of Modern Mathematics Frontier (JMMF), 2012, Vol 1 (3), $10-26$.

[4] J.H. Bae and W.G. Park, A functional equation orginating from quadratic forms, J. Math. Anal. Appl. 326 (2007), 1142-1148.

[5] P.W. Cholewa, Remarks on the stability of functional equations, Aequationes Math., 27 (1984), 76-86.

[6] S. Czerwik, Functional Equations and Inequalities in Several Variables, World Scientific, River Edge, NJ, 2002.

[7] P. Gavruta, A generalization of the Hyers-Ulam-Rassias stability of approximately additive mappings, J. Math. Anal. Appl., 184 (1994), 431-436.

[8] M. Eshaghi Gordji, H. Khodaie, Solution and stability of generalized mixed type cubic, quadratic and additive functional equation in quasi-Banach spaces, arxiv: 0812. 2939v1 Math FA, 15 Dec 2008.

[9] M. Eshaghi Gordji, M. B. Savadkouhi, Stability of Mixed Type Cubic and Quartic Functional Equations in Random Normed Spaces, Journal of Inequalities and Applications, doi:10.1155/2009/527462. 
[10] M. Eshaghi Gordji, M. Bavand Savadkouhi, Choonkil Park, Quadratic-Quartic Functional Equations in RN-Spaces, Journal of Inequalities and Applications, doi:10.1155/2009/868423.

[11] M. Eshaghi Gordji, S. Zolfaghari, J. M. Rassias and M. B. Savadkouhi, Solution and Stability of a Mixed type Cubic and Quartic functional equation in Quasi-Banach spaces, Abstract and Applied Analysis, Volume 2009, Art. ID 417473, 1-14, Doi:10.1155/2009/417473.

[12] D.H. Hyers, On the stability of the linear functional equation, Proc.Nat. Acad.Sci.,U.S.A.,27 (1941) $222-224$.

[13] D.H. Hyers, G. Isac,Th.M. Rassias, Stability of functional equations in several variables, Birkhauser, Basel, 1998.

[14] S.M. Jung, Hyers-Ulam-Rassias Stability of Functional Equations in Mathematical Analysis, Hadronic Press, Palm Harbor, 2001.

[15] Pl. Kannappan, Functional Equations and Inequalities with Applications, Springer Monographs in Mathematics, 2009.

[16] L. Maligranda, A result of Tosio Aoki about a generalization of Hyers-Ulam stability of additive functions- a question of priority, Aequationes Math., 75 (2008), 289-296.

[17] C. Park, J. R. Lee, An AQCQ-functional equation in paranormed spaces, Advances in Difference Equations, doi: 10.1186/1687-1847-2012-63.

[18] J.M. Rassias, On approximately of approximately linear mappings by linear mappings, J. Funct. Anal. USA, 46, (1982) 126-130.

[19] K. Ravi, M. Arunkumar and J.M. Rassias, On the Ulam stability for the orthogonally general Euler-Lagrange type functional equation, International Journal of Mathematical Sciences, Autumn 2008 Vol.3, No. 08, 36-47.

[20] K. Ravi, J.M. Rassias, M. Arunkumar, R. Kodandan, Stability of a generalized mixed type additive, quadratic, cubic and quartic functional equation, J. Inequal. Pure Appl. Math. 10 (2009), no. 4, Article 114, 29 pp.

[21] Th.M. Rassias, On the stability of the linear mapping in Banach spaces, Proc.Amer.Math. Soc., 72 (1978), 297-300.

[22] Th.M. Rassias, Functional Equations, Inequalities and Applications, Kluwer Acedamic Publishers, Dordrecht, Bostan London, 2003.

[23] S.M. Ulam, Problems in Modern Mathematics, Science Editions,Wiley, NewYork, 1964 (Chapter VI, Some Questions in Analysis: 1, Stability).

[24] T.Z. Xu, J.M. Rassias, W.X Xu, Generalized Ulam-Hyers stability of a general mixed AQCQ-functional equation in multi-Banach spaces: a fixed point approach, Eur. J. Pure Appl. Math. 3 (2010), no. 6, 1032-1047.

[25] T.Z. Xu, J.M. Rassias, M.J. Rassias, W.X. Xu, A fixed point approach to the stability of quintic and sextic functional equations in quasi- $\beta$-normed spaces, J. Inequal. Appl. 2010, Art. ID 423231, 23 pp.

[26] T.Z. Xu, J.M Rassias, W.X. Xu, A fixed point approach to the stability of a general mixed AQCQ-functional equation in non-Archimedean normed spaces, Discrete Dyn. Nat. Soc. 2010, Art. ID 812545, 24 pp. 